\title{
Removal of a Maxillary Third Molar From the Infratemporal Fossa
}

\author{
Lipa Bodner $^{\mathrm{a}, \mathrm{c}}$, Ben Zion Joshua ${ }^{\mathrm{b}}$, Max B. Puterman ${ }^{\mathrm{b}}$
}

\begin{abstract}
Displacement of maxillary third molar into the infratemporal fossa (ITF) is frequently mentioned but rarely reported. A case of dislocation of a maxillary third molar into the ITF is described. Imaging included CT scan that demonstrated the 3-D anatomical localization of the tooth. The tooth was found to be in the ITF, between the lateral wall of the maxillary sinus and the zygomatic arch. The tooth was retrieved immediately, under local anesthesia via intraoral approach, through the dislocation tract. The healing was uneventfull. The intraoral approach under local anesthesia allows removal of such a displaced tooth with minimal morbidity and is highly recommended.
\end{abstract}

Keywords: Tooth extraction; Third molar; Complication; Displacement; Ifratemporal fossa

\section{Introduction}

Teeth in the infratemporal fossa are considered rare. Iatro-

Manuscript accepted for publication December 7, 2011

\footnotetext{
a Department of Oral and Maxillofacial Surgery, Soroka Medical Center, Faculty of Health Sciences, and Ben Gurion University of the Negev, Beer-Sheva, Israel

${ }^{\mathrm{b}}$ Department of Laryngology and Head and Neck Surgery, Soroka Medical Center, Faculty of Health Sciences, and Ben Gurion University of the Negev, Beer-Sheva, Israel

${ }^{\mathrm{c}}$ Corresponding author: Lipa Bodner, Professor of Oral and Maxillofacial Surgery, Chairman, Department of Oral and Maxillofacial Surgery, Soroka University Medical Center, Faculty of Health Sciences, P.O. Box 151, Beer-Sheva 84101, Israel.

Email: lbodner@bgu.ac.il
}

doi:10.4021/jmc455w genic displacememt of an impacted maxillary third molar into the infratemporal fossa (ITF) is a frequently mentioned extraction complication, but is a rarely reported occurrence [1-5]. While some authors recommend urgeont hospitalization and immediate removal of the tooth under general anesthesia with the aid of image-intensifying cineradiography [6] others have suggested late removal via transantral or coronal approach $[1,7,8]$. The present report describes a case of a tooth displaced into the ITF, its diagnostic imaging and immediate surgical management.

\section{Case Report}

A healthy 34-year-old man was referred for consultation from a community dental clinic, one hour after an attempt to extract the third molar of the left maxilla, however the tooth "disappeared"(Fig.1). Panoramic radiograph (Fig. 2) demonstrated that the tooth is displaced superiorly toward the maxillary sinus. Axial computed tomography (CT) scan showed that the tooth is located in the ITF between the maxillary sinus and the zygomatic arch (Fig. 3). Alternatives of

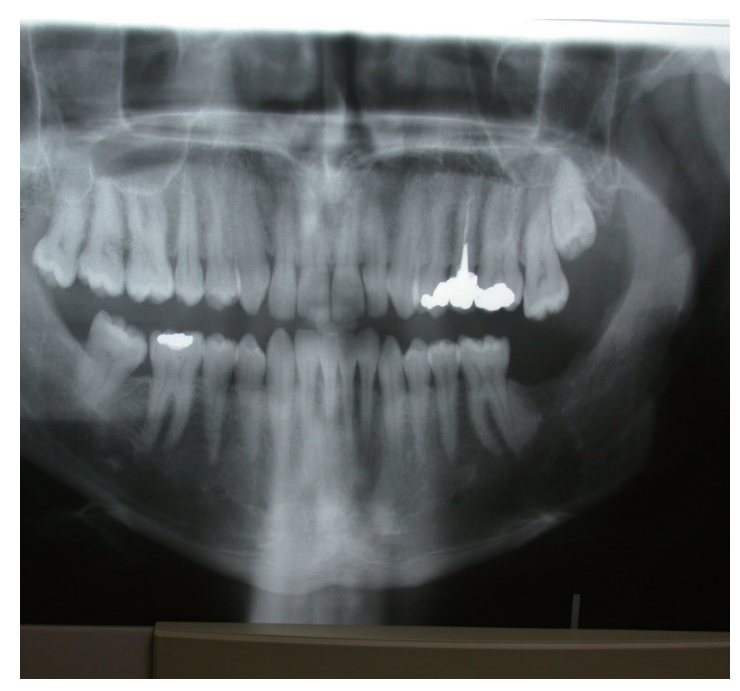

Figure 1. Preoperative panoramic radiograph. A bony impacted third molar of the Lt. maxilla can be seen. 


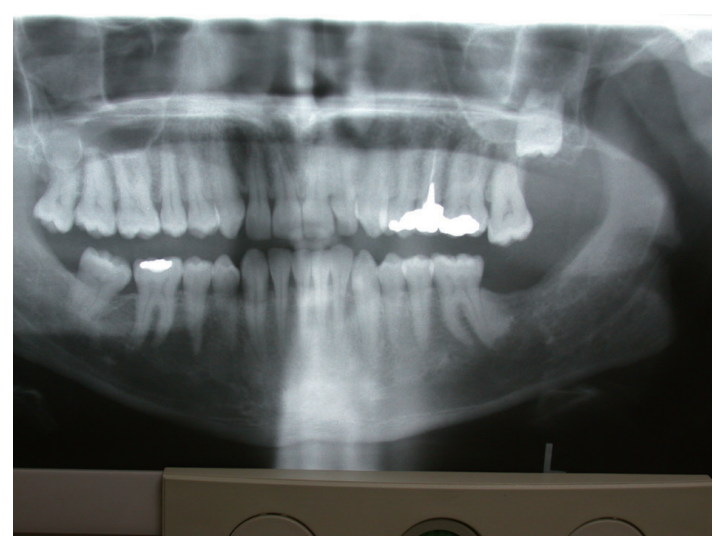

Figure 2. Panoramic radiograph showing the "disappeared" third molar displaced superiorly toward the maxillary sinus. Presently it is unclear where is the tooth in bucco-palatal plain.

treatment, either surgical or conservative were discussed with the patient, who prefered to go ahead with the surgical. Then, under local anesthesia, via the extended intraoral incision, the tooth was retrieved through the incision and the displacement tract, using a curved hemostate. Within two weeks postsurgically, the patient was fully recovered, without any adverse sequelae.

\section{Discussion}

Incorrect extraction technique or insufficient surgical training or experience are among the main reasons for displacement of maxillary third molars into the ITF. Once it is suspected, the exact anatomic location is very important to be determined radiographically. The maxillary third molars may displace either palatally, into the antrum or buccaly into the ITF.

For the radiographic work-up, panoramic, occlusal, occipitomental radiographs can be used. However, CT-scan is the most useful technique, as it provides 3-D anatomical localization [9]. Therapy attitute is based on clinical signs and symptoms, on surgeon skills and on patient decision. The complex anatomy of the ITF, the potential surgical morbidity, and the difficulty to obtain a good surgical exposure are among the limiting factors to initiate surgical treatment. However, as complications, such as infection, foreign body reaction or trismus [10], may increase if the retrieval is delayed, speaks toward immediate surgery.

On the other hand it was claimed $[3,6]$ that the displaced tooth may migrate downward into the oral cavity vestibule, allowing an easy surgical removal, which argues toward the delayed surgical approach.

Regradless the timimg of the surgery, several surgical approaches have been used succsessfuly, such as; coronal, Gillies, Caldwell-Luc or resection of the coronoid proces [4, $7,8]$. The morbidity associated with all of these approaches

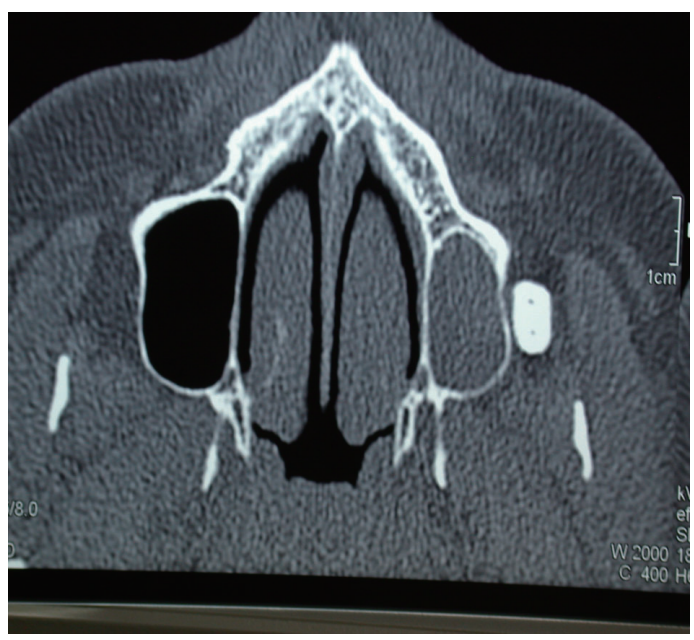

Figure 3. Axial CT scan showing the Lt. maxillary sinus full with blood and the tooth located buccally to the lateral wall of the maxillary sinus and anterior to the coronoid process. An interference to the normal anterior gliding of the coronoid process can be expected.

is high and should be taken into consideration.

The immediate intraoral approach, was chosen in the present case for the followig reasons: (1) It can be done under local anesthesia; (2) It allows the use of the displacement tract to retrive to tooth; (3) As time goes on fibrosis is developing along the displacement tract and the tooth, which makes the removal more difficult and possibly more complicated; (4) The associated morbidity is low; (5) It decreases the rate of complications.

The intraoral approach under local anesthesia allows removal of such a displaced tooth with minimal morbidity and is highly recommended. It should be used at least as the initial approach before going to other higher morbidity approach.

\section{References}

1. Winkler T, von Wowern N, Odont L, Bittmann S. Retrieval of an upper third molar from the infratemporal space. J Oral Surg. 1977;35(2):130-132.

2. Dawson K, MacMillan A, Wiesenfeld D. Removal of a maxillary third molar from the infratemporal fossa by a temporal approach and the aid of imageintensifying cineradiography. J Oral Maxillofac Surg. 1993;51(12):1395-1397.

3. Dimitrakopoulos I, Papadaki M. Displacement of a maxillary third molar into the infratemporal fossa: case report. Quintessence Int. 2007;38(7):607-610.

4. Sverzut CE, Trivellato AE, Sverzut AT, de Matos FP, Kato RB. Removal of a maxillary third molar accidentally displaced into the infratemporal fossa via intraoral approach under local anesthesia: report of a case. J Oral 
Maxillofac Surg. 2009;67(6):1316-1320.

5. Selvi F, Cakarer S, Keskin C, Ozyuvaci H. Delayed removal of a maxillary third molar accidentally displaced into the infratemporal fossa. J Craniofac Surg. 2011;22(4):1391-1393.

6. Patel M, Down K. Accidental displacement of impacted maxillary third molars. Br Dent J. 1994;177(2):57-59.

7. Orr DL, 2nd. A technique for recovery of a third molar from the infratemporal fossa: case report. J Oral Maxillofac Surg. 1999;57(12):1459-1461.
8. Gulbrandsen SR, Jackson IT, Turlington EG. Recovery of a maxillary third molar from the infratemporal space via a hemicoronal approach. J Oral Maxillofac Surg. 1987;45(3):279-282.

9. Bodner L, Tovi F, Bar-Ziv J. Teeth in the maxillary sinus--imaging and management. J Laryngol Otol. 1997;111(9):820-824.

10. Diacono MS, Wass AR. Infratemporal and temporal fossa abscess complicating dental extraction. J Accid Emerg Med. 1998;15(1):59-61. 